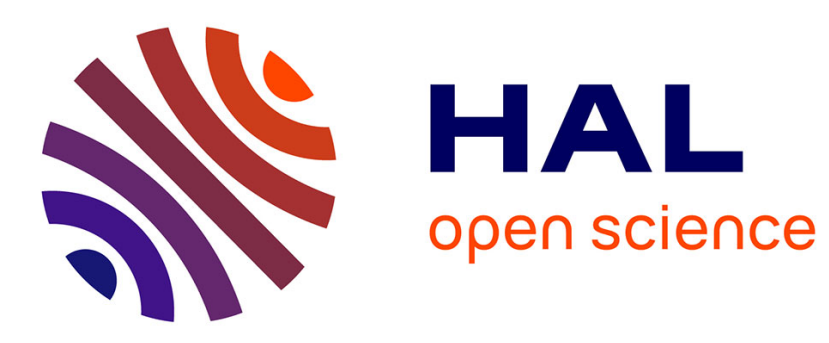

\title{
From the Classic Microscope to the Tunnel Effect Microscope
}

\author{
Michel Spajer
}

\section{To cite this version:}

Michel Spajer. From the Classic Microscope to the Tunnel Effect Microscope. Jean-Pierre Goure. Optics in Instruments, ISTE \& John Wiley, pp.267-295, 2011, 10.1002/9781118744321.ch9 . hal00598341

\section{HAL Id: hal-00598341 \\ https://hal.science/hal-00598341}

Submitted on 17 Apr 2021

HAL is a multi-disciplinary open access archive for the deposit and dissemination of scientific research documents, whether they are published or not. The documents may come from teaching and research institutions in France or abroad, or from public or private research centers.
L'archive ouverte pluridisciplinaire HAL, est destinée au dépôt et à la diffusion de documents scientifiques de niveau recherche, publiés ou non, émanant des établissements d'enseignement et de recherche français ou étrangers, des laboratoires publics ou privés. 


\title{
From the Classic Microscope to the Tunnel Effect Microscope
}

\author{
Michel SPAJER
}

\subsection{Introduction}

In this chapter we will provide an overview of the recent evolutions in optical microscopy, present in life sciences and in non-destructive testing associated with micro- and nano-technology. In terms of direction, we shall follow the notion of transfer function [SHE 92] and provide a leading role to confocal microscopy, which will allow us to comprehend it easily. For a more in-depth introduction to this discipline, we refer the reader to a number of works [GOO 70, PLU 80, ROB 99, WIL 90] as well as instructional websites recommended by laboratories and manufacturers.

The birth of modern microscopy can be traced back to the 17th Century, with the invention of the microscope, made up of an objective and an eyepiece, by an Englishman Robert Hooke (1635-1703). This gave rise to revolutionary observation of living matter (insects, plant cells). This basic structure blossomed fully in the industrial age, with the famous work of Ernst Abbe (1835-1905) who laid the theoretical foundations for the perfection of optical design. Its scientific advance is closely associated with the development of the Carl Zeiss company, in a sort of partnership which remains emblematic. The improvement of resolution, contrast, interferometry and the theory of aberrations further advanced microscopy in the $20^{\text {th }}$ Century; then, microcomputing opened up a vast range of applications for the capture and processing of images. Today, the classic optical microscope is almost 
inseperable from a camera, from the digitization of an image and from the various associated treatments. The confocal setup introduced other mechanical or acoustooptical components for scanning. The commercialization of the confocal microscope in the 1980s and the appearance of adaptive optics concretized the requirements of a diffraction limited microscopy.

The efforts to overcome the limit of resolution defined by the half-wavelength of the light involved following of two paths:

- near field microscopy, also known as local probe microscopy;

- the use of the saturation properties of certain colorants combined with the confocal microscope or structured illumination, better adapted to biological applications.

Finally, holographic microscopy aims at re-constituting the three-dimensional structure of the object without any special preparation. Widely spread structures such as living tissues gave rise to specific techniques such as OCT (Optical Coherence Tomography), which we shall not discuss in detail here.

\subsection{Towards the limit of resolution. Aspects of the formation of images}

We shall content ourselves provisionally to remain in the scalar approximation, which does not take into account the polarization of beams. It does, however, allow us to take an inventory of the problems encountered in microscopy and currently available solutions, of which we shall give a number of examples.

\subsubsection{Transfer function}

The fundamental limit of resolution or separating capability of an optical system is imposed by the diffraction, which defines the image of a point source (Point Spread Function or PSF). However, given the current aim of microscopy to reconstitute the three-dimensional structure of the object, we define the performances of the system by the normalized three-dimensional PSF, obtained by layering of images on different planes neighboring the focal plane. The relation between the object and the image is expressed by a linear relation in the two extreme cases of coherent illumination and totally incoherent illumination. The system is completely described by the modulation transfer function (MTF) of the spatial frequencies, which, in these cases, is the Fourier transform of the PSF. 


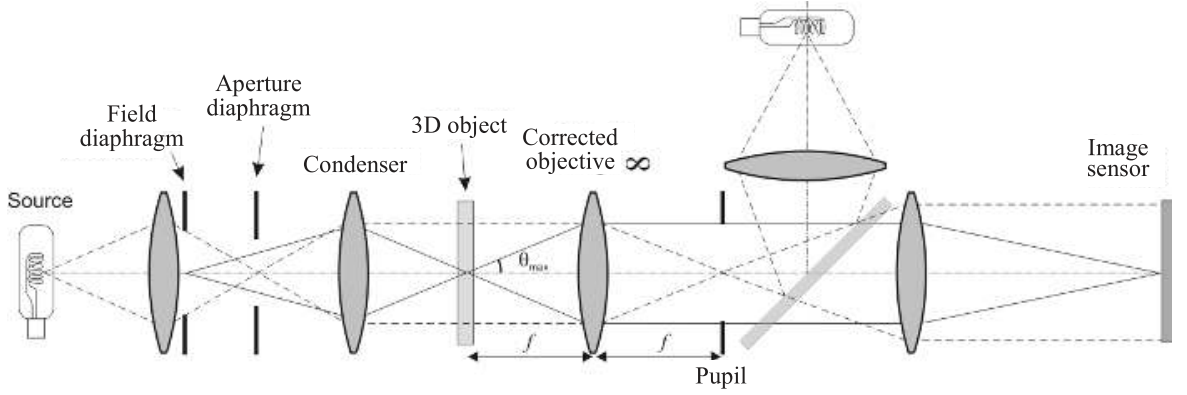

Figure 9.1. Diagram of a microscope in transmission and reflection

To explain its properties, we introduce:

- the decomposition of illumination into plane waves, where each wave is characterized by its propagation vector:

$$
\vec{k}_{s}=\frac{2 \pi n}{\lambda} \vec{u}_{s}
$$

$n$ being the index of the object environment. Spatially coherent illumination is made up of a single wave, generally in a direction parallel to the axis of the system. The extended source used in full field microscopes provides illumination which is partially coherent. Within the confines of this chapter we shall not discuss the temporal coherence of the source, which we believe is quasi-monochromatic;

- the decomposition of a three-dimensional object into a succession of superimposed grids or sinusoidal strata, with each one being characterized by its period $p$ or the spatial frequency $1 / p$ and its wave vector:

$$
\vec{K}= \pm \frac{2 \pi}{p} \vec{u}
$$

- the creation of diffracted waves according to the composition of the wave vectors:

$$
\vec{k}=\vec{k}_{s}+\vec{K}
$$

The diffracted beams passing through the edges of the aperture define the cut-off frequency of the microscope. 


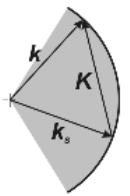

Composition of wave vectors in transmission

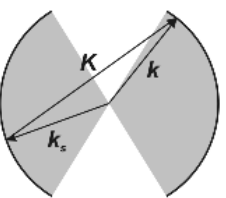

Composition of wave vectors in reflection
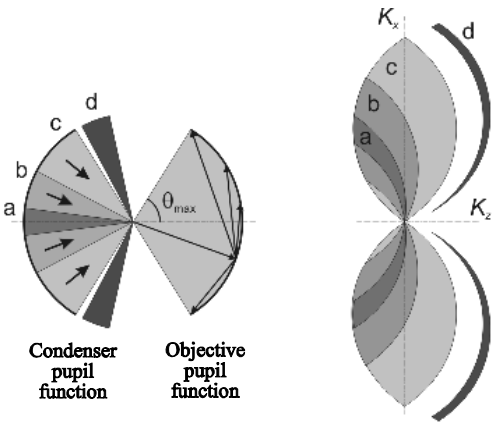

OTF support in transmission
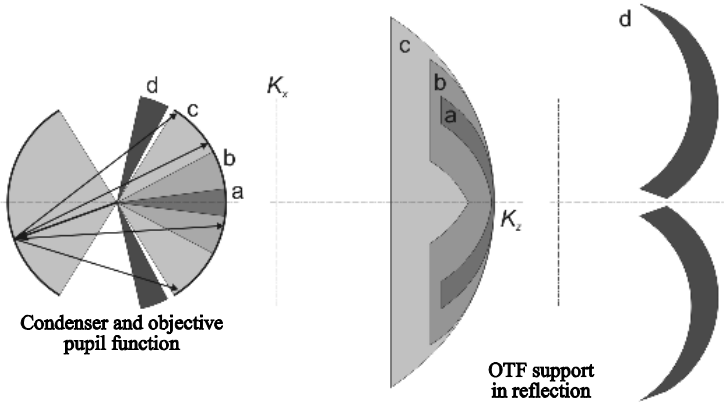

Figure 9.2. Transfer function support

This vectorial construction allows us to define the three-dimensional support of the transfer function [STR 85], in transmission as well as in reflection. Figure 9.2 shows the shape of this support for a numerical aperture of 0.85 and different values of aperture of the condenser: this is a figure of revolution, of which we will only show the cross-section. We indicate the composition of the wave vectors in transmission and in reflection, and mark in different colors the apertures $(a, b, c)$ of the condenser and the corresponding MTF supports. Strictly speaking, these figures should be completed by their symmetrical counterparts in relation to the plane $\left(\mathrm{k}_{\mathrm{x}}, \mathrm{k}_{\mathrm{y}}\right)$, given the real nature of the diffractive structures. With a slightly open condenser (a), the solution remains fairly close to the spherical cap of coherent illumination. We return to case (c) where the condenser and the objective have the same aperture: MTF is then richer in spatial frequencies. Even in this case, for medium apertures, MTF in transmission has a blind cone at certain spatial frequencies: visualization is only possible if the object contains structures whose orientation is fairly close to the $(x, y)$ plane. However, in reflection, visualization is only possible for structures of a certain thickness, excepting reflective structures. The darkest figure (d) represents the case of "dark field" observation, where none of the illuminating beams enter into the pupil of the objective: the transfer function contains no low frequency. We can similarly describe an alternative where the pupil would be ring-shaped, containing a central screen to block out directly transmitted light. 


\subsubsection{Transfer function in coherent illumination}

According to the diagrams in Figure 9.2, spatially coherent illumination corresponds to a unique direction of illumination of vector $\vec{k}_{s}$. The complex amplitude $\boldsymbol{c r}$ of the CPSF is then the 3D Fourier transform of the pupil function, i.e. the complex amplitude $\Pi(\vec{k})$ defined on the spherical cap of the same aperture as the objective, and whose beam is equal to the vector of propagation of the light:

$$
|\vec{k}|=\frac{2 \pi n}{\lambda}
$$

The conjugated variables of the $\mathrm{x}, \mathrm{y}, \mathrm{z}$ coordinates of $\vec{r}$ the object space are the components of the vector $\vec{k}$, which can also be written in cylindrical coordinates:

$$
k_{x}=\frac{2 \pi n}{\lambda} \sin \theta \cos \Phi, k_{y}=\frac{2 \pi n}{\lambda} \sin \theta \cos \Phi, k_{z}=\frac{2 \pi n}{\lambda} \cos \theta
$$

$\mathrm{NA}=n \sin \theta_{\max }$ defines the numerical aperture of the objective.

The transformation between the two amplitudes is written:

$$
c(\vec{r})=\iiint \pi(\vec{k}) \exp j \vec{k} \cdot \vec{r} d^{3} k
$$

The stigmatism condition or sine condition, established by Abbe in 1872, also regulates the relation of proportionality between the angles of aperture in object space and image space for a given magnification $G$ :

$$
\sin \left(\theta^{\prime}\right)=\sin (\theta) / G
$$

For microscope objectives, often corrected for infinity, the condition becomes, if $f$ is the focal length of the objective:

$$
\sin (\theta)=h / f
$$

If we are interested in thick samples whose structures are not very diffractive, so as to avoid multiple diffusions (Born approximation), the object-image relation is linear and is expressed simply by a convolution between the structure of the object, represented by a distribution of transparency $o(\vec{r})$, and the device function $c(\vec{r})$. In Fourier's space, the spectrum of spatial frequencies of the object $\tilde{O}(\vec{k})$ is multiplied by the FTC $\tilde{C}(\vec{k})$ to give the spectrum $A(\vec{k})$ of the image. This is summed up by the equation: 


$$
\begin{aligned}
& a(\vec{r})=\left[\exp \left(j \overrightarrow{k_{s}} \cdot \vec{r}\right) \cdot o(\vec{r})\right] \otimes c(\vec{r}) \Leftrightarrow \\
& A(\vec{k})=\left[\tilde{O}(\vec{k}) \otimes \delta\left(\vec{k}-\overrightarrow{k_{s}}\right)\right] \cdot \Pi(\vec{k})=\tilde{O}\left(\vec{k}-\overrightarrow{k_{s}}\right) \cdot \Pi(\vec{k})
\end{aligned}
$$

This describes a convolution or a product between the complex amplitudes and transparency, taking into account the phase shifts introduced by the object or the pupil. We have attempted to indicate the role of the incident wave by the first exponential term, but this expression is founded on the over-simplified hypothesis that the distribution of transparency is independent of the incident light. We shall see the relevance of this in the framework of holography, outside of which coherent illumination is of little interest, as the interference between two neighboring points is the noise factor. The pupil function $\Pi(\vec{k})$ must be uniform across the whole aperture, but it can have a non-uniform phase if the objective suffers from aberrations. This is accessible by monitoring the objective with a Twyman-Green interferometer. The intensity of the PSF will be of use to us in the framework of incoherent illumination.

\subsubsection{Aberrations}

The entire art of a manufacturer consists of realizing the sine condition in the whole field of the objective, and at all the wavelengths used. The difference from this condition is described in terms of the difference from the ideal wave surface (Chapter 1). It is developed in the form:

$$
\Delta=\Sigma \mathrm{a}_{\mathrm{pnm}} h^{\mathrm{p}} \rho^{\mathrm{n}} \cos \mathrm{m}\left(\Phi+\Phi_{0}\right)
$$

where $h$ is the height of the object in relation to the axis of the system, with $\rho$ and $\Phi$ the polar coordinates on the pupillary plane.

The best known are defocalization $\left(\rho^{2}\right)$, and first-order aberrations: spherical aberration $\left(\rho^{4}\right)$, coma $\left(h \rho^{3} \cos \Phi\right)$, astigmatism $\left(h^{2} \rho^{2} \cos ^{2} \Phi\right)$, curvature of field $\left(h^{2} \rho^{2}\right)$, distortion $\left(h^{3} \rho \cos \Phi\right)$. Chromatic aberration (dependence on the focusing with the wavelength) must be added to this list, which it is vital to compensate for, in order to avoid an iridescent blurring of the images.

The correction of these errors is important for the calculation of all optical combinations, from astronomy to microscopy. The correction of the spherical aberration must take into account the thickness of the cover slip, between $0.17 \mathrm{~mm}$ and $0.2 \mathrm{~mm}$. Manufacturers specify the objective of the thickness of cover slip for which it is adjusted. The widest open objectives can even have an adjustable ring to adapt them to different thicknesses. It is, however, difficult to perfectly correct an objective for the entire field. We shall see that the adaptive optics associated with 
the confocal microscope has found a solution to this problem. Figure 9.3 shows the deformation of the PSF for differences limited to $\lambda$.
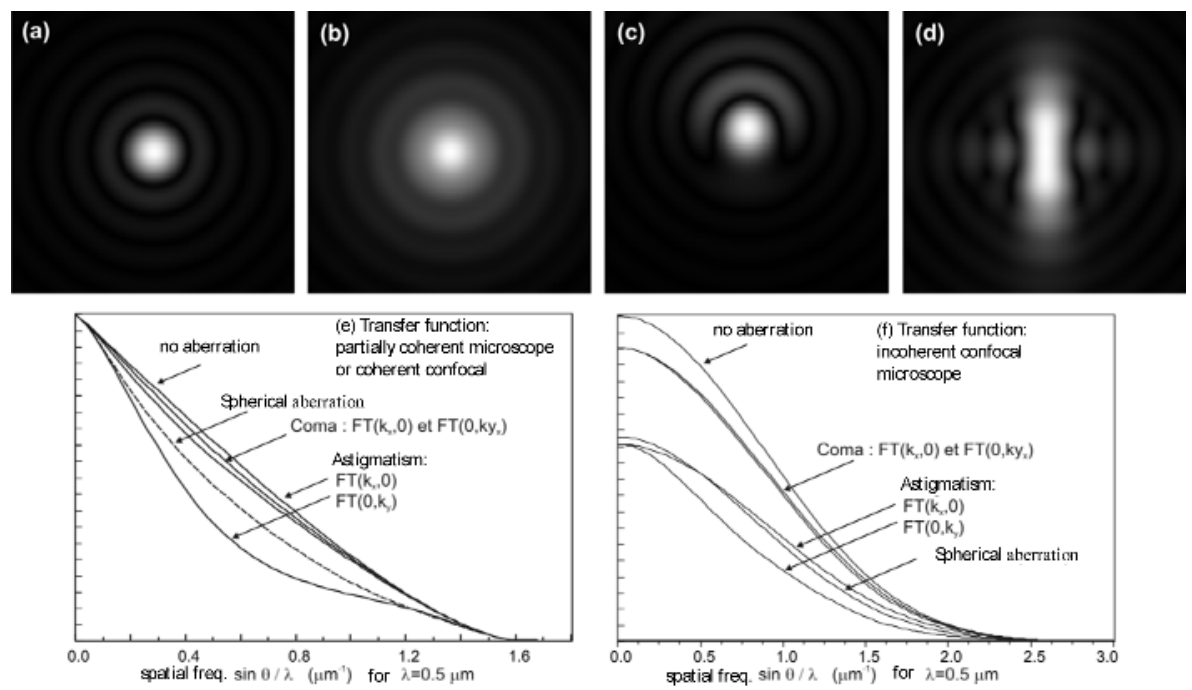

Figure 9.3. Influence of aberrations $\left(\Delta_{\max }=2 \pi\right)$ on focalization and transfer function. Focal spot: (a) with no aberration, (b) spherical aberration,

(c) coma, (d) astigmatism, (e-f) transfer function based on the type of microscope

\subsubsection{Transfer function in partially coherent illumination}

Illumination is said to be partially coherent when two very close emitting points of the object have a phase relation, so that their contributions are added in amplitude on the image. To clarify this situation, we use the notion of mutual intensity which defines the visibility of interference between the emissions of the two points:

$$
\Gamma\left(\vec{r}_{1}, \vec{r}_{2}\right)=\iint S(\vec{k}) \exp \left[-j\left(\vec{r}_{1}-\vec{r}_{2}\right) \cdot \vec{k}\right] d^{2} k
$$

where $\vec{r}_{1}$ and $\vec{r}_{2}$ are the positions of the two points and $\vec{k}$ the wave vector corresponding to the points of the secondary source $\mathrm{S}_{\mathrm{s}}$. This function of the distance between the two points is none other than the Fourier transform of the distribution of intensity in an extended source (Van Cittert-Zernike theorem). We can show [COU 87] that the spectrum of intensity in the image is of the form:

$$
\tilde{I}(\vec{k})=\iint T\left(\vec{k}+\overrightarrow{k^{\prime}}, \overrightarrow{k^{\prime}}\right) \tilde{O}\left(\vec{k}+\overrightarrow{k^{\prime}}\right) \tilde{O}^{*}\left(\overrightarrow{k^{\prime}}\right) d^{2} k
$$


which involves the function $T\left(\vec{k}, \overrightarrow{k^{\prime}}\right)$, called a bilinear transformation, which depends not only on the pupil function but on the function $S$ :

$$
T\left(\vec{k}, \overrightarrow{k^{\prime}}\right)=\iint S\left(\vec{k}_{s}\right) \Pi\left(\vec{k}+\vec{k}^{\prime}+\vec{k}_{s}\right) \Pi^{*}\left(\vec{k}+\vec{k}_{s}\right) d^{2} k_{s}
$$

It involves the surface which is common to the three disks $S\left(\vec{k}_{s}\right)$, $\Pi\left(\vec{k}+\overrightarrow{k^{\prime}}+\vec{k}_{s}\right)$, and $\Pi\left(\vec{k}+\overrightarrow{k^{\prime}}+\vec{k}_{s}\right)$. This result will not be detailed here. If the angular extension of the source is much greater than that of the two pupils ( $S\left(\vec{k}_{s}\right)$ constant), we find ourselves in the limiting case of incoherent illumination and $T\left(\vec{k}, \overrightarrow{k^{\prime}}\right)$, is the autocorrelation of the pupil function, as we shall see in section 1.2.5. This case occurs only with a moderate pupil aperture.

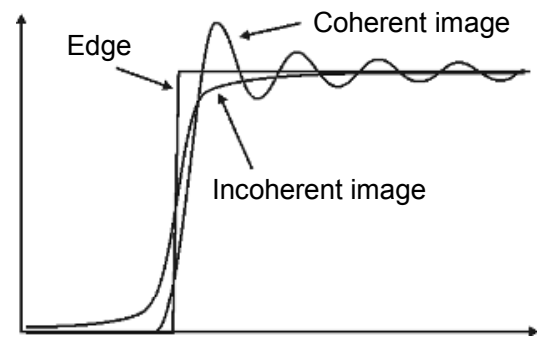

Figure 9.4. Images of a screen edge

Every user of a microscope must choose the spatial coherence of the illumination. The microscope carries out two conjugations (Figure 9.1): that of the field stop with the object and that of the aperture stop with the focal image plane of the objective. This configuration, advocated in around 1893 by August Köhler, is always respected. We observe that by reducing to a minimum the aperture stop (the source becomes almost a point or spatially coherent), we reinforce the contrast of the edges of objects, to the detriment of resolution. However, by opening this diaphragm to the maximum (illumination aperture $=$ objective aperture), the resolution is optimized, but the contrast of the edges or the finer details is low. The best compromise is found when the illumination aperture is around $70 \%$ of the objective aperture. These observations show that resolution depends as much on the conditions of illumination as on the imaging system. We shall see the manner in which it has been put to use in recent decades to overcome the limits of resolution.

Metrology depends to a great extent on these conditions of illumination, which must be perfectly known for a correct interpretation of the images to be possible. Figure 9.4 gives an example of this with the image of a metallic boundary. 


\subsubsection{Transfer function in incoherent illumination}

The best example of totally incoherent illumination is fluorescence microscopy: the object is considered as luminous itself, as we only take into account the incoherent light (no phase relation between two neighboring points) re-emitted by fluorescent markers. Under these conditions, it is the intensities of the different emitters which is added on the image plane and not the amplitudes, which is expressed by:

$$
\begin{aligned}
& i(\vec{r})=i_{0}(\vec{r}) \otimes\left[c(\vec{r}) \cdot c^{*}(\vec{r})\right] \\
& \Leftrightarrow \tilde{I}(\vec{k})=\tilde{I}_{0}(\vec{k}),\left[\Pi(\vec{k}) \otimes \Pi^{*}(\vec{k})\right]=\tilde{I}_{0}(\vec{k}) \cdot T_{i}(\vec{k})
\end{aligned}
$$

where $i_{o}(r)$ is the intensity emitted by each point of the object. The incoherent transfer function $(I T F)$ is the Fourier transform of the incoherent PSF $|c(\vec{r})|^{2}$, therefore the auto-correlation of the pupil function:

$$
T_{i}(\vec{k})=\Pi(\vec{k}) \otimes \Pi^{*}(\vec{k})
$$
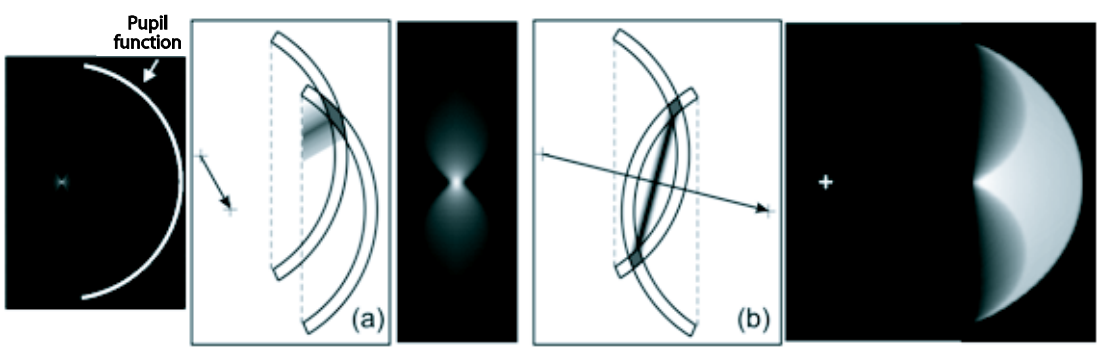

Figure 9.5. Construction of the transfer functions of an incoherent full field microscope in transmission or reflection (a) and of a coherent confocal microscope in transmission (a) and reflection (b)

Figure 9.5a gives a graphic representation of this auto-correlation. We have deliberately given a certain thickness to the spherical cap, assuming that the light is not completely mono-chromatic, which corresponds to fluorescence imaging. The auto-correlation $T_{\mathrm{i}}(\vec{r})$, coded here in gray level, is proportional to the volume common to both caps separated by the shift $\vec{r}$, normalized by its value $T_{\mathrm{i}}(\overrightarrow{0})$, is sometimes modified by the phase of the pupil function. In the case of an almost monochromatic light, this volume leans toward 0 but the normalized function $T_{\mathrm{i}}(\vec{r}) / T_{\mathrm{i}}(\overrightarrow{0})$ tends toward a finite limit. For very small apertures $(N A<0.2)$, we 
practically find the well-known autocorrelation function. More generally, if we are interested in the component of spatial frequencies parallel to the focal plane, the associated transfer function is obtained by integration along the $z$ axis of the $3 \mathrm{D}$ function.

The central lobe of the 3D PSF constitutes the diffraction ellipsoid. It is accessible by imaging a fluorescent particle of nanometric dimensions, and layering the images obtained on different focal planes. The transfer function can be obtained numerically by the Fourier transform of the PSF or by auto-correlation of the pupil function obtained by interferometry. The section of the 3D PSF by the plane $z=0$ is a series of rings surrounding a central peak whose radial distribution of intensity is the Airy curve (Figure 9.6):

$$
I(r)=\left[\frac{J_{1}\left(3.83 r / r_{0}\right)}{3.83 r / r_{0}}\right]^{2} \text { with } r_{0}=1.22 \frac{\lambda}{2 O N}=1.22 \frac{\lambda}{2 n \sin (\vartheta \max )}
$$

In object space, the radius of the first dark ring $\mathrm{r}_{0}$ gives us the lateral resolution of the microscope according to the aperture of the objective, which is always provided by the manufacturer. We should underline the relevance of increasing the index $n$ of the object space in order to increase the resolution (immersion objectives). As for the axial resolution, it is given by the distribution of intensity on the $z$ axis:

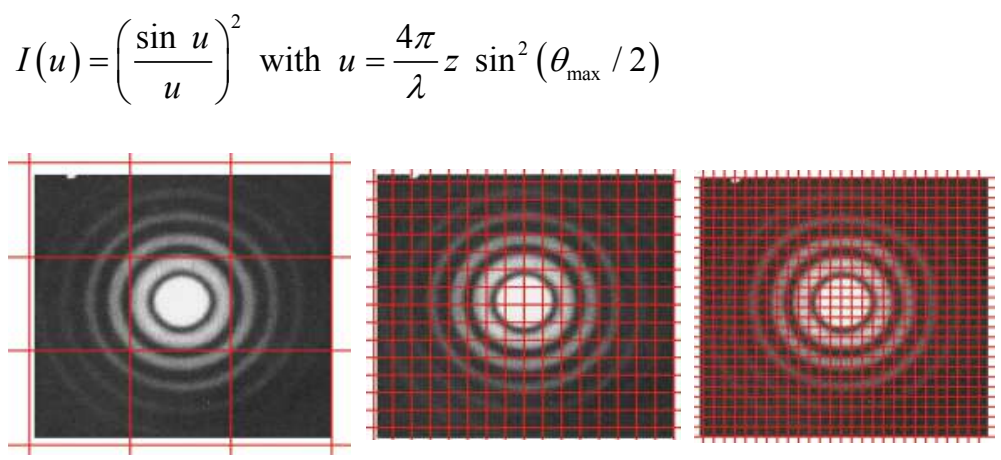

Figure 9.6. Sampling by a digital image sensor (under-sampling, adapted sampling, over-sampling)

We must associate the digital resolution with these physical limits, which is linked to the size of the pixels of the camera. In order to benefit from the performances of the objectives, this must be, at most, equal to half the diffraction 
spot (Nyquist's sampling criterion). Over-sampling is sometimes necessary, for example to measure the PSF, or in interferometric microscopy.

In incoherent illumination, the extension of the illumination aperture allows higher spatial frequencies, to diffract beams toward the objective aperture: when the illumination aperture is equal to the objective aperture, the cut-off frequency is then twice as great. This statement must be qualified, as the small portion of the light which is involved in imaging for this frequency means that the contrast is almost null. The effective cut-off frequency can also be lowered by the level of noise of the sensor.

\subsubsection{Structured illumination, synthetic pupil}

We have seen how the conditions of illumination influenced the resolution. Particular conditions can even lead, in fluorescence, to super-resolution, beyond the limit of $\lambda / 2 \sin \theta_{\max }$ imposed by the numerical aperture: this involves structured illumination, with spatial frequencies which are equal to or greater than the cut-off frequency of the system. It allows us to access high frequencies of the object thanks to the moiré phenomenon, which in practical terms means expanding the pupil function of the system. This operation uses digital processing of the data, hence the notion of the synthetic pupil [GUS 00].

This is summarized in Figure 9.7: the object chosen for simplicity of demonstration emits a fluorescence modulated by a deformed grid (a), not resolved by the microscope. The spectral components of this object are indeed situated beyond the aperture (b) and the image (c) contains no information. If the illumination is modulated by a known regular grid (d), only the moire figure is visible in the image (j) once its spectral components (i) are within the pupil. The phase shifting method, now widely used in interferometry as well as in moiré here, allows us to define at each point of the image the module and phase of the moiré figure (1): they are deduced from the four images recorded with four positions of the illumination grid, a quarter period apart. The Fourier transform of this complex image and of the conjugate image are then shifted by the frequency $1 / \mathrm{p}$ of the illumination grid (n). The inverse Fourier transform reconstructs the object (m). This procedure completes the pupil of the system with two pupils shifted by $1 / \mathrm{p}$. For any fluorescent object which can be decomposed into a superimposition of grids of all orientations, the complete treatment of an image necessitates following this procedure for at least three orientations of the grid, so that twelve images must be combined. The synthetic pupil represented as a dotted line in (n) then has a satisfying degree of symmetry. 


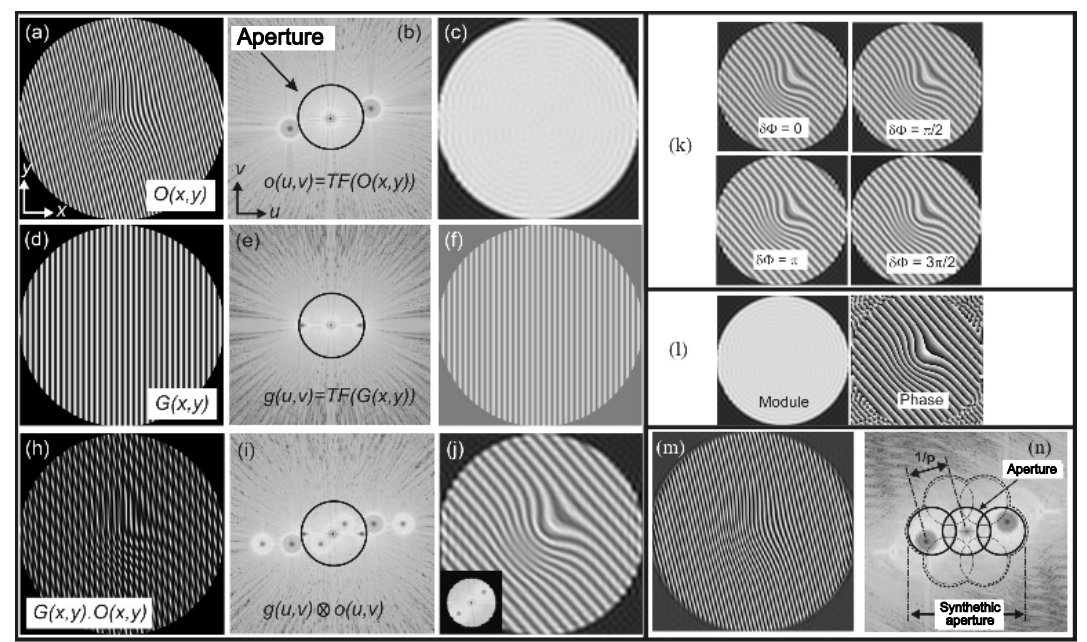

Figure 9.7. Incoherent (fluorescence) microscopy, with structured illumination

The cut-off frequency of the image can be increased still further by playing on the non-linearity by saturation of the fluorescence emission [GUS 04]. Figure 9.8 illustrates the distribution of the emission in two illumination conditions (a): when we attain the conditions of saturation of the fluorescence, the image becomes richer in spatial harmonics (b) and the synthetic pupil obtained extends in proportion to the number of significant harmonics (c). In order to maintain an almost circular symmetry in the resulting pupil, we must here record 24 images corresponding to six orientations of the grid.
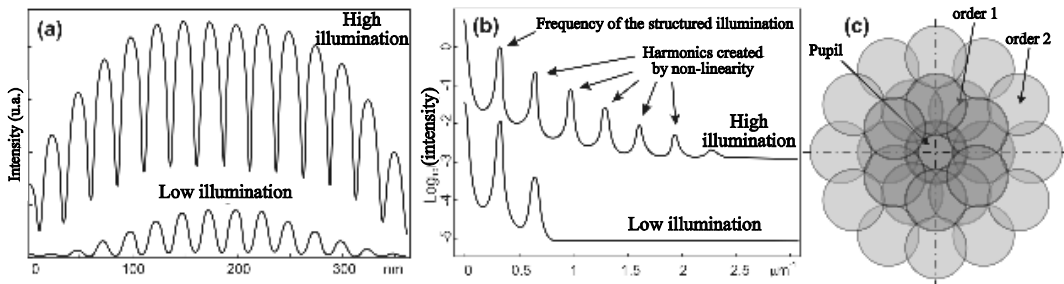

Figure 9.8. Structured illumination and fluorescence saturation. (a) Distribution of fluorescence; (b) spectrum of the image; (c) synthetic pupil obtained with twelve orientations of the illumination grid, and limited to the two first orders

\subsection{The confocal microscope}

The reflection confocal microscope, the principle of which is shown by Figure 9.9, works by scanning the object by the image of a micro-aperture. The light 
re-emitted by the object is detected through the same aperture, which plays the role of a filtering hole. By rapid scanning, the multiple apertures of a spinning disk allow simultaneous observation of several points on the object. They are far enough apart, so that the diffraction spots of two points lit at the same time do not overlap. The change in intensity detected according to the position $\mathrm{z}$ of the surface is treated in parallel for all the points by a camera and a specialized electronic unit. Other scanning systems are used: galvanometric mirrors or acousto-optical modulators, especially with laser sources used in fluorescence, or a mechanical translation of the object, notably in profilometry. A transmission system is less often used, as it involves synchronizing the receptive aperture and the emitting aperture.

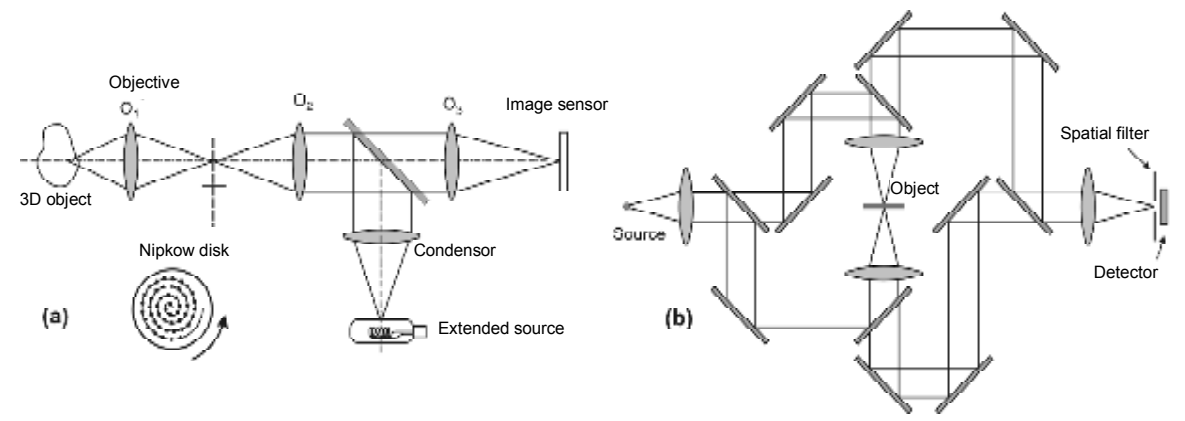

Figure 9.9. (a) Principle of the reflection confocal microscope and (b) $4 P i$

The PSF can again be obtained by scanning a simple object made up of a reflective or fluorescent micro-particle of nanometric dimensions. Then we must distinguish the coherent function, which corresponds to 3D imaging of a surface and the incoherent function, corresponding to fluorescence.

\subsubsection{Coherent confocal microscope}

Let us consider (Figure 9.10) all the points $M(\vec{r})$ lit by the objective's diffraction ellipsoid, characterized by the amplitude distribution $c_{1}(\vec{r})$. Each point $P(\vec{r})$ of the image space receives the sum of the contributions in amplitude of its neighboring points, which is expressed by the convolution:

$$
a(\vec{r})=\iiint c_{1}(\vec{r}) c_{2}\left(\overrightarrow{r^{\prime}}-\vec{r}\right) d^{3} r^{\prime}
$$

If $P$ belongs to the filtering aperture and this is far smaller than the limit of resolution $(\mathrm{OP} \sim 0)$, then the coherent point spread function (CPSF) in amplitude is: 


$$
c(\vec{r})=c_{1}(\vec{r}) c_{2}(-\vec{r})
$$

$c_{1}(\vec{r})$ and $c_{2}-\vec{r}$ are complex amplitudes, Fourier transforms of the pupil functions $\Pi_{1}(\vec{k})$ and $\Pi_{2}^{*}(\mathrm{k})$. The transfer function is thus similar to that of a full field microscope in incoherent illumination:

$$
T_{c}(\vec{k})=\Pi_{1}(\vec{k}) \otimes \Pi_{2}^{*}(\vec{k}) x
$$

It is represented by Figure 9.5a. In reflection, the supports of the pupil functions $\Pi_{1}$ and $\Pi_{2}$ are symmetrical in relation to the xy plane. Their inter-correlation and the result of it are represented by Figure $9.5 \mathrm{~b}$.
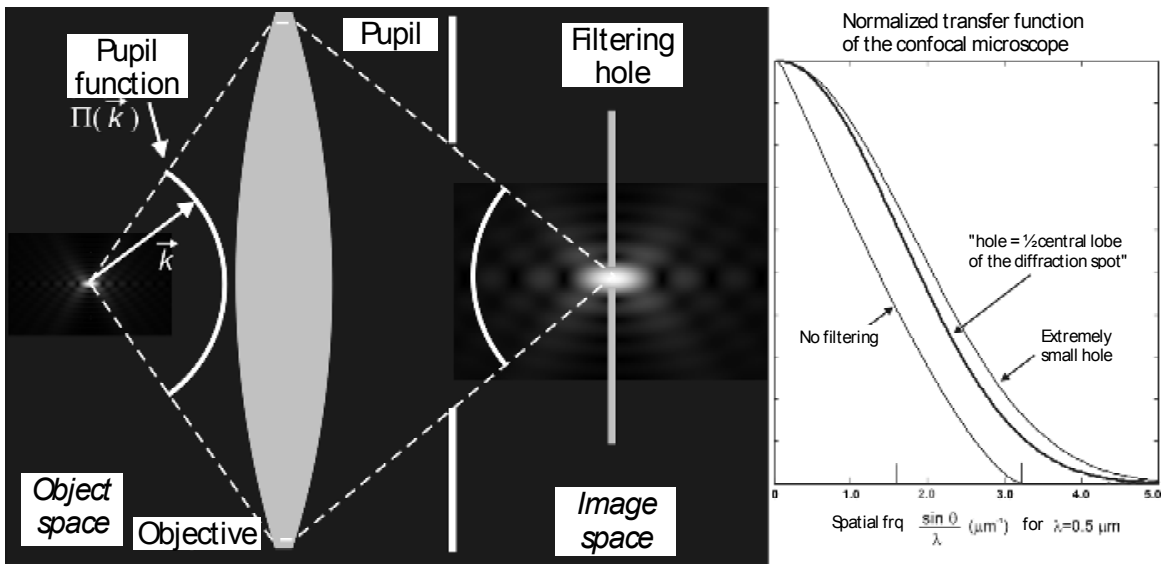

Figure 9.10. Formation of the image in confocal microscopy. In the object space the diffraction ellipsoid has been juxtaposed with the pupil function, which belongs to the conjugate space. In the image space, the image spot (which a point lit by the object ellipsoid produces) is truncated by the filtering hole.

\subsubsection{Incoherent confocal microscope (fluorescence)}

In the case of fluorescence, the different points lit by the diffraction ellipsoid in turn emit different contributions whose intensities, and not the amplitudes, are added together in the image. This is expressed by the convolution:

$$
i(\vec{r})=\iiint i_{1}(\vec{r}) i_{1}\left(\overrightarrow{r^{\prime}}-\vec{r}\right) d^{3} r^{\prime}
$$


Indeed, there is no more reason to distinguish transmission from reflection: they will have the same transfer function. The intensity received by an aperture of finite dimension can here be integrated more simply than in the case of a coherent microscope:

$$
\begin{aligned}
& i_{F}(\vec{r})=\iint d^{3} r^{\prime} \iiint i_{1}(\vec{r}) i_{1}\left(\overrightarrow{r^{\prime}}-\vec{r}\right) d^{3} r=\iiint i_{1}(\vec{r}) i_{2}(-\vec{r}) d^{3} r \\
& \text { with } i_{2}(\vec{r})=\iint_{h o l e} i_{1}\left(\vec{r}+\overrightarrow{r^{\prime}}\right) \mathrm{d}^{3} \mathrm{r}^{\prime}=i_{1}(\vec{r}) \otimes f(\vec{r}) \\
& \text { where } f(\vec{r})=0 \text { if }|\vec{r}|>r_{0}, f(\vec{r})=1 \text { if }|\vec{r}| \leq r_{0}
\end{aligned}
$$

$f(\vec{r})$ is the transparency of the filtering hole and $\tilde{F}(\vec{k})$ its Fourier transform. The DF is then the function:

$$
i(\vec{r})=i_{1}(\vec{r}) \cdot i_{2}(-\vec{r})
$$

and the incoherent transfer function:

$$
T_{i}(\vec{k})=\left[\Pi_{1}(\vec{k}) \otimes \Pi_{1}^{*}(\vec{k})\right] \otimes\left\{\left[\Pi_{1}(\vec{k}) \otimes \Pi_{1}^{*}(\vec{k})\right] \cdot F(\vec{k})\right\}
$$

It should be noted that in the absence of filtering $\left(r_{0}=\infty\right)$, the OTF (and the PSF) is identical to that of a classic full field microscope or to the CTF of the confocal microscope. The technique of scanning loses its interest, except when we manage to reduce the dimensions of the PSF, as we shall see later on.

If, on the other hand, the diameter of the hole is far smaller than the central lobe of the diffraction spot, $\tilde{F}(\vec{k})$ is considered to be uniform, the PSF is the square of the CPSF: the central lobe becomes smaller. The OTF is the auto-correlation of the CTF: the transversal and axial cut-off frequencies are doubled. However, the OTF is continuous in the region of the 0 frequency: we can image a fluorescent plane with no transversal structures, which a classic microscope cannot do.

In practical terms, the filtering hole must be of finite dimension, so as not to degrade the signal-noise ratio. The OTF is then intermediary between the two previously mentioned functions. Figure 9.10 compares their values for the curve obtained when the radius of the hole is equal to $r_{0} / 2$, which appears to be an acceptable value. 
The PSF can again be obtained by using several successive focal planes to scan a simple object made up of a reflective or fluorescent micro-particle of nanometric dimensions.

\subsubsection{Pi synthetic aperture}

A fundamental limitation in imaging 3D object is the difference between the longitudinal and lateral resolutions. We also know that two counter-propagating mutually coherent beams, create a standing wave, with a constructive interference where the two optical paths are identical. The central peak is $\lambda / 2$ in width. Thus, two confocal beams of large aperture, but in opposite directions have the same diffraction ellipsoid but their interference produces a longitudinal modulation of this ellipsoid, whose central peak can become the new PSF of the system. The situation is therefore reversed: the longitudinal dimension benefits from the ultimate resolution of $\lambda / 2$. This functionality imposes very precise adjustments to cancel out optical delays, between incident beams as well as among beams re-emitted by the object. Figure 9.11 sums up the advantages an incoherent confocal microscope has over a coherent confocal microscope, and which the 4Pi confocal microscope has over the classic confocal microscope. The numerical aperture, although it is great, no longer allows continuity in the transfer function. The elimination of this residual modulation gets rid of the lateral lobes of the PSF (Figure 9.11e).

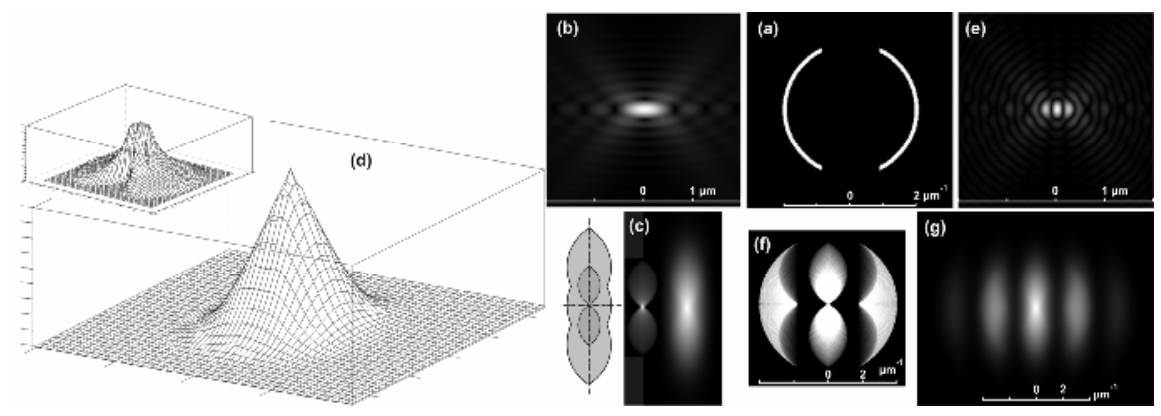

Figure 9.11. (a) Resolution of the confocal microscope and the 4Pi confocal microscope. Pupil function of the 4Pi confocal microscope; (b) PSF of a single one of the pupils;

(c) coherent and incoherent transfer functions (d) and their profiles; (e) PSF of the 4 Pi confocal microscope and $(f)$ its coherent or incoherent (g) transfer function

Here we come to the problem of de-convolution, the final digital stage for improving the image, as it appeared implicitly in formulae [9.20] and [9.26]: we could ideally find the spectrum of the object by dividing that of the image by the 
transfer function, previously obtained from a Fourier transform of the PSF. An inverse Fourier transform would therefore restore a de-convoluted image of the object. This operation (obtaining a uniform transfer function) is not that easy, since the measured transfer function contains zeros and the image is degraded by noise. Figure 9.11e allows us to define a more realistic ambition, which consists, by eliminating the lateral lobes of the PSF, to reduce it to its central lobe. The final OTF is then an intermediary between the ideal OTF and the OTF of the raw image. In practical terms, the de-convolution of images is done by iterative algorithms which we cannot describe in detail here.

The principle of double illumination and double observation has been applied to full field microscopy [GUS 99] with the same advantages.

\subsubsection{Stimulated emission depletion (STED) confocal microscope}

We have seen how the non-linearity of the emission of fluorescence can improve the resolution. An even more radical variation of two-photon excitation microscopy was developed from the 1990s onward, to achieve resolutions of a few tens of nanometers, which we call "STED" (Stimulated Emission Depletion) [HEL 00, $\mathrm{SCH}$ 06]. It consists of reducing the diameter of the emissive zone by excluding emission on the periphery of the focal spot by previous illumination by a ringshaped "inhibitor" beam (Figure 9.12).
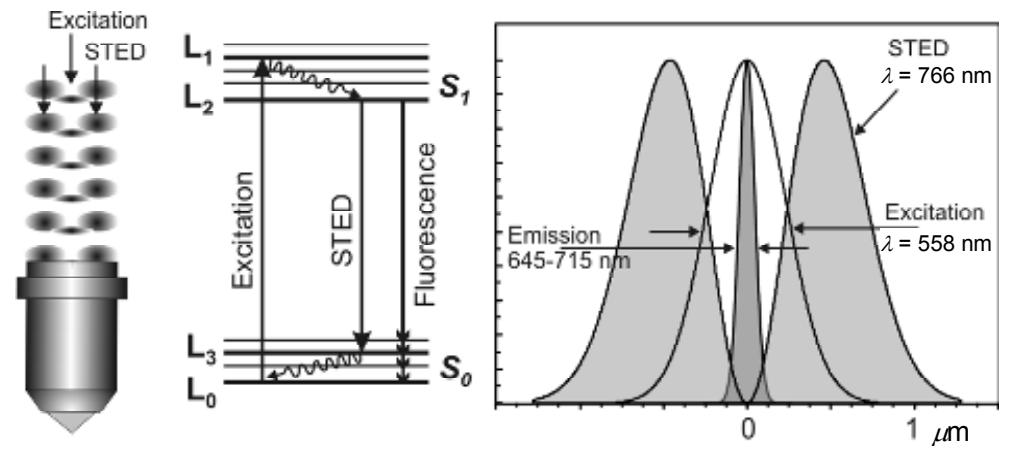

Figure 9.12. Principle of the STED microscope: structure of the pulse trains, electronic levels of the colorant (Styryl), focal spot of the inhibitor, excitation and emitted beams

We must use the properties of the quantum levels of colorant: $\mathrm{S}_{0}$ and $\mathrm{S}_{1}$ represent the base level and the excited level, $\mathrm{L}_{0}$ being the lowest vibrational sub-level of $\mathrm{S}_{0}$ and $L_{1}$ the vibrational sub-level directly excited by the excitation pulse. $L_{2}$ is the level attained by relaxation of $\mathrm{L}_{1}$ and $\mathrm{L}_{3}$ is the upper level of $\mathrm{S}_{0}$. The role of the 
inhibitor beam (STED) is to induce the $\mathrm{L}_{2} \rightarrow \mathrm{L}_{3}$ transition by stimulated emission to empty the excited level and thus prevent the emission of fluorescence. It is modulated into a train of pulses (typically $70 \mathrm{MHz}$ ) whose duration (typically $40 \mathrm{ps}$ ) is greater than the relaxation time of the $\mathrm{L}_{1}$ level (typically $0.2 \mathrm{ps}$ ). The pulse train (typically $0.2 \mathrm{ps}$ ) of the excitation beam is temporally shifted from the former, so that the excitation occurs at the end of the STED pulse. Since the inhibition effect is non-linear, a sufficiently powerful (typically $2.8 \mathrm{GW} / \mathrm{cm}^{2}$ ) ring-shaped beam can reduce the emissive part of the focal stain to a diameter, far smaller than the wavelength. Lateral resolutions of $35 \mathrm{~nm}$ have been achieved. The STED was combined with 4Pi bi-directional illumination, which brings the axial resolution to similar values [SCH 06]. This research, mainly carried out at the Max Planck Institute, constitutes an important advance in providing the confocal microscope with a spherical 3D PSF of around $\lambda / 20$ in size.

\subsection{Adaptive optics}

Whatever the quality of the objectives, the wave-front is deformed by passing through a biological medium, generally in-homogeneous. As in astronomy, this deformation can be compensated for in real time thanks to a piezo-deformable mirror, so that the spot focused on the sample regains its quality of being limited only by diffraction.

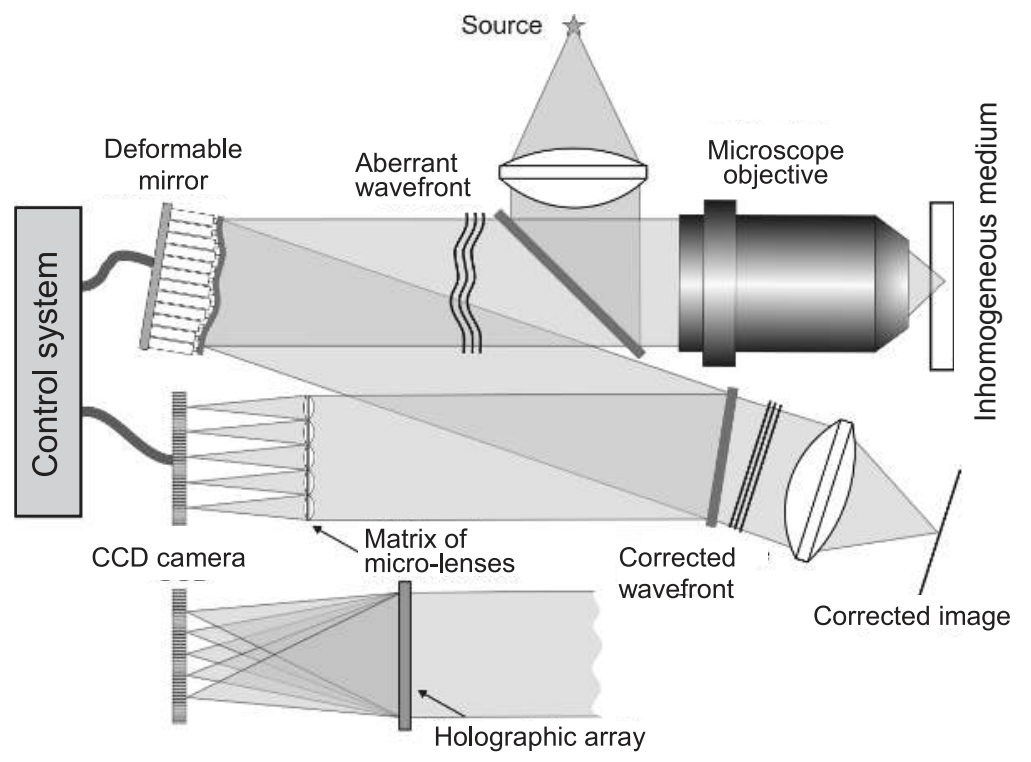

Figure 9.13. Principle of adaptive optics 
The control often uses the Shack-Hartmann method, which consists of sampling the wave-front with the help of a matrix of micro-lenses, each component beam being focused on a CCD image sensor (Figure 9.13). When the wave-front is perfect, the focal stains are circular and regularly spaced. The aberrations of the medium displace and deform the component stains. An algorithm calculates the deformations to be given to a mirror to compensate these errors. The quality of the system is thus linked to the rapidity of interfacing of the piezo-electric switches.

This method is not always easily usable in microscopy, where the image rarely contains a "point source" on which the control is based. However, the confocal microscope can put its scanning and focusing procedures to good use to optimize the signal at each point. Also, deformations due to the medium, unlike the atmospheric disturbances present in astronomy, can generally be described by third-order terms, which consist of as many "modes" of deformation. An iterative procedure allows us to discover the weight of each mode in the deformation of the wave and finally correct it [BOO 07].

\subsection{Polarized light}

Our description of the PSF and transfer function remains valid in paraxial conditions, for objectives with small apertures (in practice up to NA < 0.5). Wide apertures necessitate a vectorial approach more in tune with physical reality; if the illumination beam (or the beam emitted by the object), is linearly polarized, the PSF cannot have symmetry of revolution. The state of polarization for the different rays of the beam depends on the refractions imposed by the diopters of the objective. Let us take as an example an ideal convergent beam, initially polarized according to the $x$ axis (electrical field $E_{x}$ ) whose polarization would remain linear upon refraction (absence of phase-shifting between the $E_{r}$ and $E_{\theta}$ component polar coordinates). The Cartesian components of the electrical field in the convergent beam become (Figure 9.11):

$$
\begin{aligned}
& e_{x}=E_{x}\left(\cos \theta \cos ^{2} \Phi+\sin ^{2} \Phi\right) \\
& e_{x}=E_{x} \cos \Phi \sin \Phi(\cos \theta-1) \\
& e_{z}=E_{x} \sin \theta \cos \Phi
\end{aligned}
$$

The PSF regains symmetry of revolution with non-polarized light, but the central maximum is widened. The polarizing properties of very high-N.A beams cannot be reduced to an inconvenience for resolution: they can for example, be used to detect the orientation of the fluorescent molecules assimilatable to a dipole. We are no 
longer limited to beams which are uniformly polarized in a linear or circular fashion: we can take advantage of ring-shaped beams with ortho-radial or radial polarization [BEV 06]. With the latter, it is the longitudinal component $E_{z}$ of the focal spot which contains a central maximum and which can be preponderant in image formation.

Independently of these properties of beams of wide aperture, polarization, very early on, opened a well-known range of applications in the observation of birefringent materials: crystals, but also organic fibers.
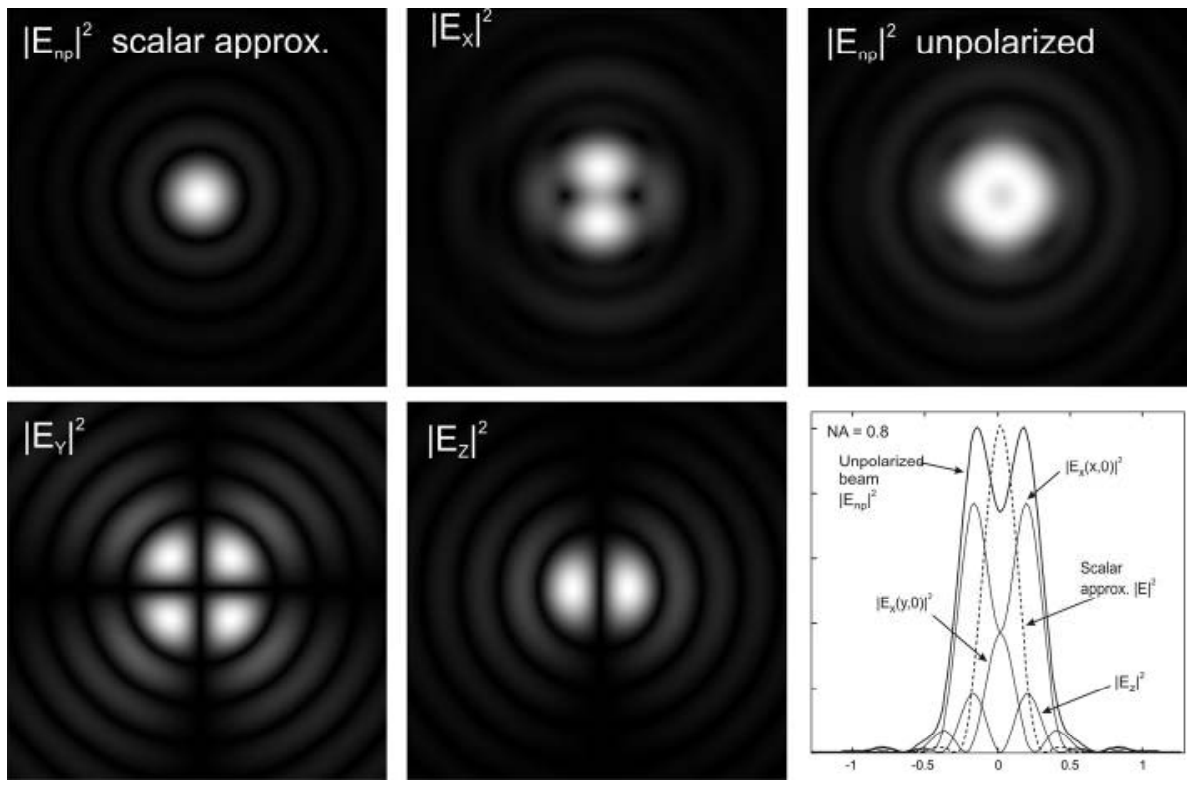

Figure 9.14. Magnitude of polarization at wide apertures

\subsection{Phase microscopies}

In the previous section, the phase parameter of electromagnetic field has not yet been used, other than to establish the relation between resolution and wave-front. Microscopy effectively uses methods of interferometry but imposes certain constraints on them, particularly linked to aperture. Among the great advances in microscopy, we must list phase contrast, introduced in 1934 by Zernike and differential interference contrast (DIC) by Nomarski. Here we shall limit ourselves to some recent developments with a view to giving a quantitative interpretation of interferometric data or to three-dimensional imagery. 


\subsubsection{Absolute interferometric phase-shifting measurements}

Phase contrast is based on the phase shift $\pi / 2$ introduced between the light diffracted by a slightly diffusing object and the transmitted light. A phase plate introduced onto the Fourier plane allows us to compensate it and thus make the diffracted light interfere with the direct beam, which allows us to contrast the slight phase variations. The phase modulators which have appeared in recent decades allow us to quantify these variations in phase, drawing on the phase shifting method [POP 08]. The biological object is lit by a laser diode of very slight coherence (Figure 9.15).

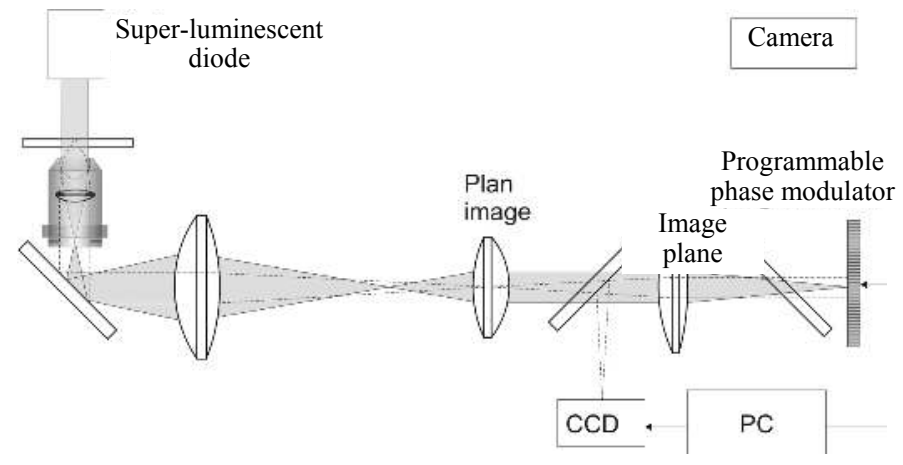

Figure 9.15. Fourier transform phase microscopy

The Fourier transform (or spatial spectrum) of the image, after passing through a polarizer, is projected onto a liquid crystal programmable phase modulator. A monitoring camera allows us to align the spectrum on the modulator. The modulator allows us to introduce a controlled phase shift between the diffracted light and the directly transmitted beam. We can then carry out a measurement by phase shifting, which is described later. The processing of the image is only limited by the frame rate of the modulator, which can be up to $1 \mathrm{kHz}$. The advantage of this type of setup is its high stability (10 to $20 \mathrm{mrad})$.

An alternative consists of separating the reference pathway and the measurement pathway: it is very widely used in profilometry [CRE 08]. The reflection setup indeed allows compact set ups which are not very sensitive to parasitic vibrations. Figure 9.16 shows several types of interferometers combined with a microscope objective. A Michelson setup can be placed in front of the object, as long as the working distance allows us to place the separating cube between them, which prohibits large magnification. For expansions such as $\times 100$, it is essential that the geometric and chromatic aberrations be identical on both pathways of the 
interferometer. This leads to the use of two carefully matched objectives placed on each arm (Linnick setup) at the same distance from the separator. In front of the $\times 40$ magnification objectives, at a long working distance, we can place a separating strip focusing the reference beam towards a reflective treatment localized on the first lens (Mirau objective). This setup is particularly stable and reliable.
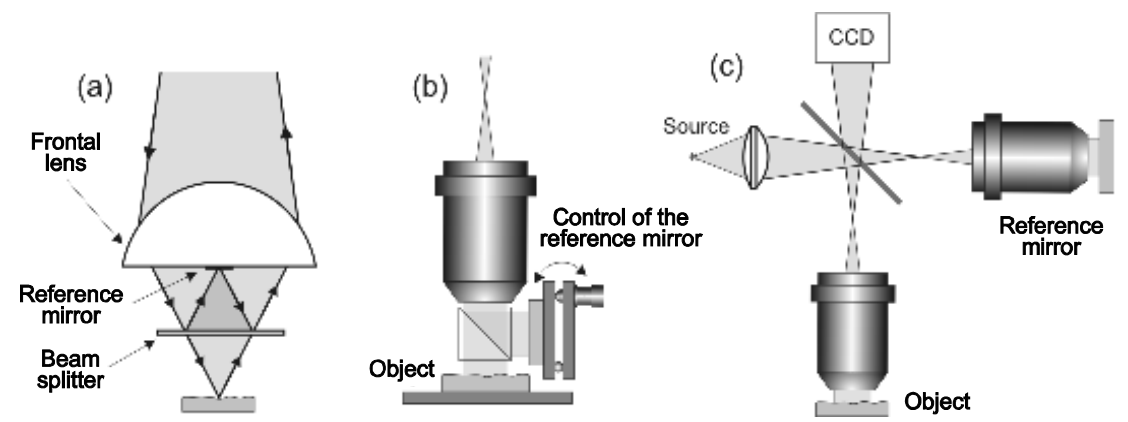

Figure 9.16. (a) Interference phase-shift microscopy: Mirau objective, (b) Michelson interferometer and (c) Linnick interferometer
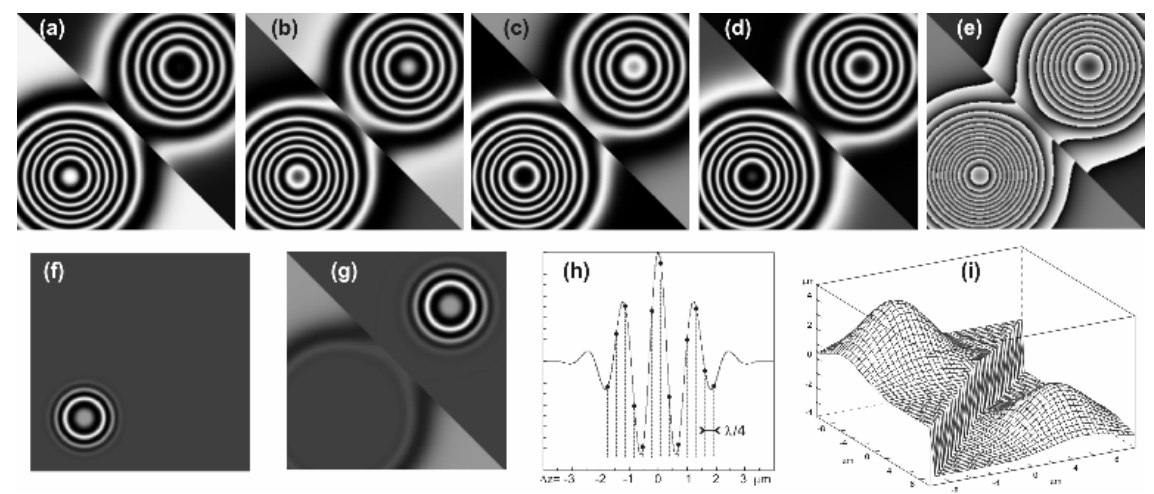

Figure 9.17. (a-d) Phase shifting in coherent light: four interferograms shifted by $\pi / 2$, (e) calculation of the phase, $(f-g)$ Measurement in incoherent light: focusing on two areas of the object, (h) correlogram sampling at each pixel, (i) reconstitution of the relief of the object

We use coherent light (a laser or spectral lamp equipped with a narrow chromatic filter) when the reliefs of the surface being studied are slight (a few wavelengths) and do not contain any step which would make the interpretation of the fringes ambiguous. The phase-shifting method consists of combining several interferograms recorded by incrementing the phase shift between the beams by moving vertically the objective controlled by a piezo-electric translator. Four interferograms shifted by 
$\pi / 2$ are most often used (Figure 9.17a-d) but other combinations can offer a better tolerance for errors of calibration. The very simple algorithm giving the phase at each point is then:

$$
\Phi(\mathrm{x}, \mathrm{y})=\operatorname{arctg}\left\{\left[\mathrm{I}_{2}(\mathrm{x}, \mathrm{y})-\mathrm{I}_{4}(\mathrm{x}, \mathrm{y})\right] /\left[\mathrm{I}_{1}(\mathrm{x}, \mathrm{y})-\mathrm{I}_{3}(\mathrm{x}, \mathrm{y})\right]\right\}
$$

However, when the surface of the object contains "steps", as in our example, the calculation of phase cannot remove ambiguities linked to discontinuity (Figure 9.17e). This situation is common in micro-systems. The use of white or not very coherent light allows us to remove any ambiguity, since only the points whose altitudes are below a few wavelengths give rise to contrasted interferences (Figure $9.17 \mathrm{f}-\mathrm{g}$ ). The complete study of the topography implies a larger movement of the objective and the treatment of a greater number of interferograms than before. At each pixel of the image, the movement of the objective produces a correlogram or signal of interference surrounded by the coherence function of the source (Figure 9.17h). The sampling of this signal allows us to deduce the maximum of the correlogram and therefore the altitude of the point in question, with the help of an adapted algorithm (Figure 9.17i). The use of cameras and fast digitization cards allows us to apply these image treatments to moving objects.

\subsubsection{Measurements based on a single interferogram}

Certain situations prevent the taking of several interferograms, like non-periodic vibrations or moving objects, if we do not have ultra-fast means of treatment at our disposal. The deformation phase can be reconstructed in two ways, which use an essential property of the Hilbert transform: this transforms every sine component of a signal into a cosine, and vice versa, hence its relevance for calculating the phase when we only have the cosine modulation of a lone interferogram:

- the one-dimensional Hilbert transform [IKE 06] is a variation of the method described in section 9.2.6. By appropriate inclination of the reference beam, we can artificially add straight-line fringes to the fringes induced by the $\Phi(\mathrm{x}, \mathrm{y})$ phase of the object. $I_{R}$ and $I_{S}(x, y)$ are the intensities of the reference beam and the object beam. A high-pass filtering extracts the modulated part of the signal which, for each line of the image, is written:

$$
u(x)=2 \sqrt{I_{R} I_{s}} \cos [q x+\Phi(x)]
$$

From this we deduce the analytical signal $z(x)$, which is the sum of $u(x)$ and its Hilbert transform, from which we can extract the $\Phi(x)$ phase: 


$$
z(x)=\frac{1}{2} u(x)+i \frac{P}{2 \pi} \int_{-\infty}^{\infty} \frac{u\left(x^{\prime}\right)}{x-x^{\prime}} d x^{\prime} \quad \Phi(x)=\tan ^{-1}\{\operatorname{Im}[z(x)] / \operatorname{Re}[z(x)]\}
$$

- the spiral quadrature transform [LAR 01]. The SQT is an isotropic Hilbert transform, using a spiral function to determine the phase. It is very well adapted to phase demodulation when the interference fringes are extremely deformed, crossed or closed.

\subsubsection{D holographic microscopy}

Holography allowed us, in the 1970 s, to study the shapes and movements of an object based on recordings on photo-sensitive plates of the amplitude diffracted by the object. Digital cameras and image combination have provided an alternative means to reconstitute the three-dimensional structure of the object, particularly nearly transparent biological objects which we do not wish to label with dyes (label free microscopy) [LAU 02, SIM 08]. This involves sequential recording of images obtained in the different directions of illumination made possible by the objective or condenser being used (Figure 9.18): amplitude and phase are digitized, using the interference of a reference beam and the phase shifting technique previously described. The 3D image is then re-constructed, avoiding parasitic phase-shifting between the different component images, which is a delicate point of the method.
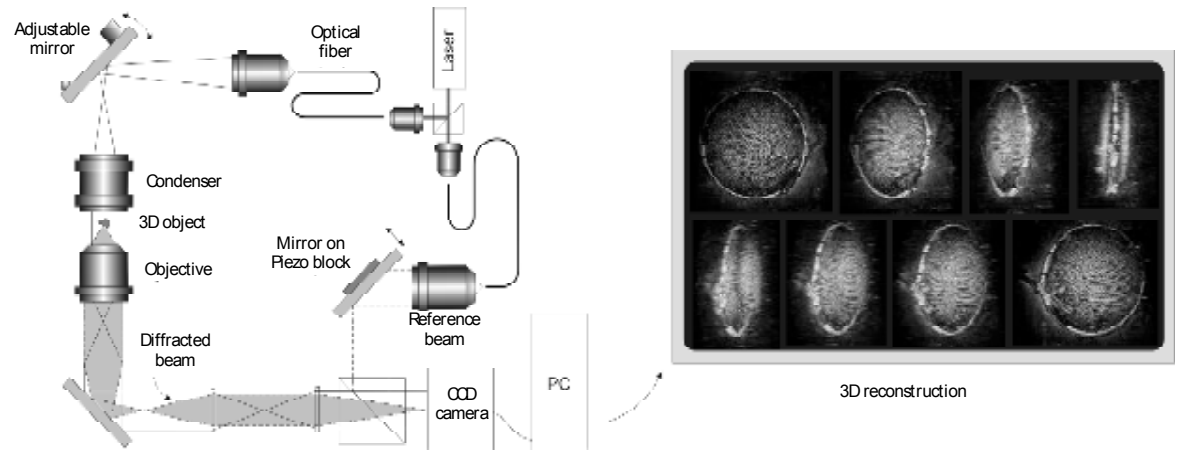

Figure 9.18. Holographic microscopy as shown in [SIM 08]

The direction of illumination (flat wave) is varied thanks to an adjustable mirror. The illumination beam is partially diffracted. The direct beam is also spread on the CCD camera: this serves to normalize, in amplitude and in phase, the different holograms recorded by phase shifting. 


\subsection{Confined light microscopy techniques. Evanescent waves}

The resolution in microscopy is limited, as we have seen, by the numerical aperture $2 \mathrm{n} \cdot \sin \theta$ of the objective, hence the importance of increasing the index of the object environment, at an unchanged angular aperture. However, without recourse to immersion, we can take advantage of the evanescent waves which accompany the total reflection in an objective, lit in super-critical conditions: they are propagated along the diopter in the low-index environment and have as a wave vector the projection of the wave vector of the reflected beam k.n. $\sin \theta$. The resolution therefore profits from the index of the glass in the lens. The depth of the structures observed is limited by the penetration of the evanescent wave into the low-index environment, of the order of $100 \mathrm{~nm}$.

An initial illustration is given by optical tunnel microscopy [GUE 90]. When we bring a rough, dielectric surface toward the front of the objective, the reflection is locally frustrated by the "summits" of the relief, which then appear in dark on a light background (Figure 9.19a). Its application is limited to dielectric objects, hence we use polymer casts to study the surface quality of metallic samples. Its sensitivity to changes in altitude is similar to that of interferometric microscopy, with a better lateral resolution.
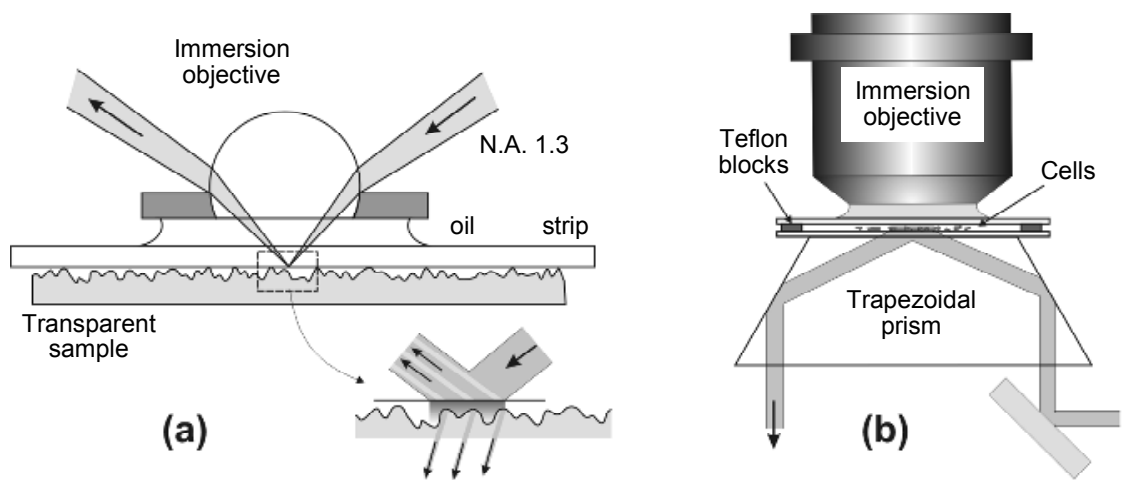

Figure 9.19. (a) Optical tunnel microscopy and (b) TIRFM

This illumination is more directly adapted to biological structures: Total Internal Reflection Fluorescence Microscopy (TIRFM) in particular allows us to eliminate parasitic light by limiting the illuminated depth (Figure 9.19b). Here, as in incoherent light (section 9.2.6), structured illumination also allows us to push the resolution to its very limits [CHU 06]. Indeed, two beams of supercritical incidence, but of opposite azimuths create an evanescent field modulated by a standing wave. The phase shift between the beams allows us to scan the lighted area with an array 
of sinusoidal fringes and view, with excellent contrast, particles that are smaller than a half-wavelength in the glass $(\lambda / 2 n)$.

\subsection{Near-field local probe microscopy}

The principle of the near-field optical microscope was suggested in 1928 by E.H. Synge before being re-invented in 1972 in the area of centimetric waves and then in the 1980s in the area of optics, at the same time as other local probe microscopes: the STM and the AFM. With the latter microscope, it shares two essential functions: scanning of the object, controlled by piezoelectric translators and keeping the probe at a distance of a few nanometers from the object by a regulation mechanism. The key element of this microscope is the probe, whose function is to capture the nonpropagative components of the electromagnetic field, which are confined to the neighborhood of a structure smaller than the wavelength. Two classic examples are the field transmitted by a nano-aperture in a metallic screen and the radiation of a dipole, which contains components whose amplitude decreases as $1 / \mathrm{r}^{2}$ and $1 / \mathrm{r}^{3}$. This field is predominant over the propagative field, decreasing as $1 / r$ when the distance $r$ is smaller than the wavelength. The probe itself is generally a nano-aperture pierced at the extremity of a dielectric metallized tip, or a metallic structure playing the role of a nano-antenna. Alternatively, it can play the role of a nano-source, scanning the structures being studied, the metallic tips being the seat of confinement of light. In both cases, the resolution is no longer linked to the wavelength, but to the dimensions of the probe extremity or to the aperture: a few tens of nanometers, or more rarely, a few nanometers. The object-image relationship cannot be described by so simple an operator as convolution, given the interaction between the probe and the nanostructures and image processing uses more sophisticated algorithms. Nanoantennas are the subject of research, which aims at mastering their polarizing properties (butterfly antennae, ring-shaped antennae, etc.). The essential difficulty lies in reconciling the technological sophistication with the robustness necessary to follow the topography of the object.

The theoretical bases and applications of near-field optics are developed in many works or monographs [COU 01, COU 03, NOV 07]. In keeping with our previous policy, we shall limit our discussion to two of the numerous configurations used. The first is the Scanning Tunneling Optical Microscope (STOM) or Photon scanning tunnel microscope (PSTM) where the object placed on a slide is lit by evanescent waves (Figure 9.20a). The advantage is in reducing the amount of direct light captured by the probe, which benefits the signal-to-noise ratio. Among its applications we can cite the study of polymers, cellular structures, light confinement and the plasmon propagation in metallic structures. 


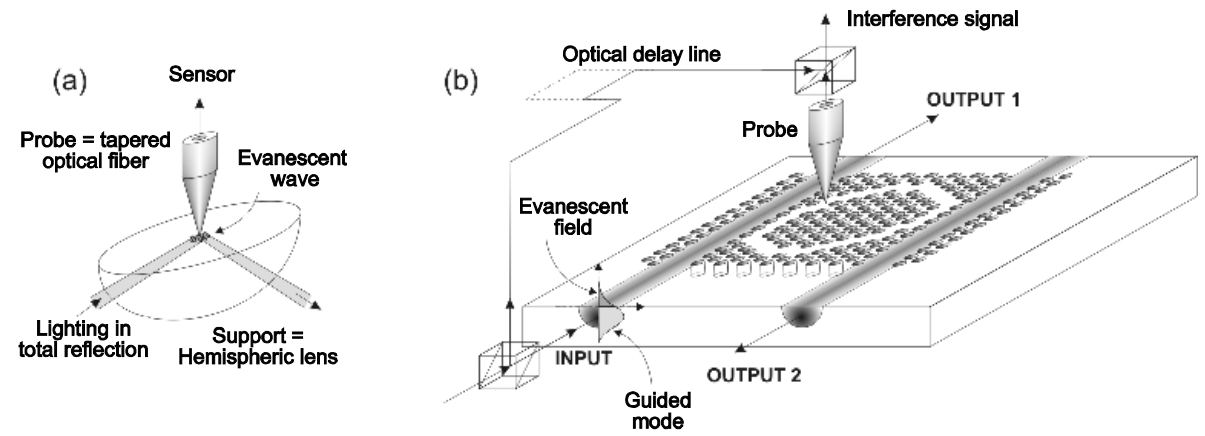

Figure 9.20. (a) Near-field scanning microscopy: tunnel microscope, (b) scanning heterodyne microscope

Evanescent waves also accompany the propagation of modes in guiding structures. They can then be studied, as they allow us to characterize the properties of the guide. Indeed, a probe scanning the surface of the guide absorbs a very small fraction of the guided mode energy transported by its evanescent part, without disturbing it detectably. Heterodyne interferometry can also measure the phase of the mode by combining the light captured by the probe with a reference beam shifted in frequency. This allows us to access modal dispersion in complex, dielectric or plasmonic structure, in continuous or pulsed mode. This device also allows us to study the propagation of light in photonic crystals (Figure 9.20b).

Another relevant application concerns fluorescence and local spectroscopy. The sub-wavelength confinement of light in the neighborhood of the exciting probe allows us to carry out finer spectroscopy of molecules according to their physical ("quenching" in the neighborhood of metallic surfaces) and chemical environment. It also allows the spectroscopy of isolated molecules with lesser dilutions than in classic microscopy.

\subsection{Bibliography}

[BEV 06] Beversluis M.R., Novotny L., Stranick S.J., "Programmable vector pointspread function engineering", Optics Express, vol. 14, p. 2650-2656, 2006.

[BOO 07] Воотн M.J., "Adaptive optics in microscopy", Philosophical Transactions of the Royal Society A, vol. 365, p. 2829-2843, 2007.

[CHU 06] Chung E., Kim D., So P.T., "Extended resolution wide-field optical imaging: objective-launched standing-wave total internal reflection fluorescence microscopy", Optics Letters, vol. 31, p. 945, 2006. 
[COU 87] Courjon D., Charraut D., Livrozet P., "Bilinear transfer in microscopy", Journal of Modern Optics, vol. 34, p. 127-136, 1987.

[COU 01] COURJON D., BAINIER C. (ed.), "Le champ proche optique. Principes et applications", Springer-Verlag France and France-Telecom R\&D, Paris, 2001.

[COU 06] CourJon D., "Near-field microscopy and near-field optics", Imperial College Press, London, 2006.

[CRE 08] CREATH K., "Phase-shifting interferometry techniques", in E. Wolf (ed.), Progress in Optics XXVI, p. 357-373, Elsevier Science Publishers, Amsterdam, 2008.

[GOO 05] Goodman J.W., Introduction to Fourier Optics, Roberts \& Company Publishers, Englewood, 2005.

[GUE 90] Guerra J.M., "Photon tunneling microscopy", Applied Optics, vol. 29, p. 3741-3752, 1990.

[GUS 99] Gustafsson M.G.L., Agard D.A., Sedat J.W., "I5M: 3D widefield light microscopy with better than $100 \mathrm{~nm}$ axial resolution", Journal of Microscopy, vol. 195, p. 10-16, 1999.

[GUS 00] GUSTAFSSON M.G.L., "Surpassing the lateral resolution limit by a factor of two using structured illumination microscopy”, Journal of Microscopy, vol. 198, p. 82-87, 2000.

[GUS 04] GUSTAFSSON M.G.L., "Nonlinear structured-illumination microscopy: Wide-field fluorescence imaging with theoretically unlimited resolution", Proceedings of the National Academy of Sciences of the United States of America, vol. 102, p. 13081-13086, 2004.

[HEL 00] Klar T.A., Jakobs S., Dyba M., Egner A., Hell S.W., "Fluorescence microscopy with diffraction resolution barrier broken by stimulated emission", Proceedings of the National Academy of Sciences of the United States of America, vol. 97, p. 8206-8210, 2000. This group's publications are accessible on the website of the Max Planck Institute: www.mpibpc.mpg.de/groups/hell/.

[IKE 05] Ikeda T., Popescu G., Dasari R.R., Feld M.S., "Hilbert phase microscopy for investigating fast dynamics in transparent systems", Optics Letters, vol. 30, p. 1165-1167, 2005.

[LAR 01] LARKIN K.G., Bone D.J., OldFiEld M.A., "Natural demodulation of twodimensional fringe patterns. I. General background of the spiral phase quadrature transform", Journal of the Optical Society of America A, vol. 18, p. 1862-1870, 2001, "Stationary phase analysis of the spiral phase quadrature transform", Journal of the Optical Society of America A, vol. 18, p. 1871-1881, 2001.

[LAU 02] LAUER V., "New approach to optical diffraction tomography yielding a vector equation of diffraction tomography and a novel tomographic microscope", Journal of Microscopy, vol. 205, p. 165-176, 2002.

[NOV 07] Novotny L., "The History of Near-field Optics", in E. Wolf (ed.), Progress in Optics, vol. 50, chap. 5, p.137-184, Elsevier, Amsterdam, 2007. The publications of the Nano-Optics group of the University of Rochester can be consulted on the site: www.optics.rochester.edu/workgroups/novotny/ 
[PLU 89] PluTa M., Advanced Light Microscopy, Elsevier, PWN - Polish Scientific Publishers, Warsaw, 1989.

[POP 08] POPESCU G., "Quantitative phase imaging of nanoscale cell structure and Dynamics", Methods in Cell Biology, vol. 90, chap. 5, p. 87-114, Elsevier Science Publishers, Amsterdam, 2008.

[ROB 99] RoBlin G., "Microscopie optique”, Techniques de l'Ingénieur, R6712, 10 June 1999.

[SCH 06] Schmidt R., Wurm C.A., JakOBS S., Engelhardt J., Egner A., Hell S.W., "Spherical nanosized focal spot unravels the interior of cells", Nature Methods, vol. 5, p. 539-544, 2006.

[SHE 90] Sheppard C.J.R., CogSwell C.J., "Three-dimensional image formation in confocal microscopy", Journal of Microscopy, vol. 159, p. 179-194, 1990.

[SHE 92] SHEPPARD C.J.R., MiN GU, "The significance of 3-D transfer functions", Journal of Microscopy, vol. 165, p. 377-390, 1992.

[SIM 08] Simon B., Debailleul M., Georges V., Lauer V., Haeberlé O., "Tomographic diffractive microscopy of transparent samples", The European Physical Journal Applied Physics, vol. 44, p. 29-35, 2008.

[STR 85] STREIBL N., "Three-dimensional imaging by a microscope", Journal of the Optical Society of America A, vol. 2, p. 121-127, 1985.

[WIL 90] Wilson T. (ed.), “Confocal microscopy”, Academic Press, London, 1990.

\subsection{Glossary of terms used}

Pupil function: $\Pi(\vec{k})$

Coherent point spread function (CPSF): $C(\vec{r})$

Coherent transfer function (CTF): $\tilde{C}(\vec{k})$

Incoherent point spread function (PSF): $i(\vec{r})$

Incoherent optical transfer function (OTF): $\tilde{I}(\vec{k})$

Coordinates in object space: $\vec{r}=x \vec{u}_{x}+y \vec{u}_{y}+Z \vec{u}_{z}$

Coordinates in Fourier space: $\vec{k}=k_{x} \vec{u}_{x}+k_{y} \vec{u}_{y}+k_{z} \vec{u}_{z}$

Integrals: $\iiint d^{3} r=\iiint d x d y d z, \iiint d^{3} k=\iiint d k_{x} d k_{y} d k_{z}$

$$
\delta\left(\vec{k}-\overrightarrow{k_{s}}\right)=1 \text { if } \vec{k}=\vec{k}_{s}, \delta\left(\vec{k}-\overrightarrow{k_{s}}\right)=0 \text { if } \vec{k} \neq \vec{k}_{s}
$$

Fourier Transform (FT)

Numerical Aperture (NA) 